\title{
The Exploratory Research of NSCLC with Concomitant EGFR Mutations and ALK Rearrangements
}

\section{Qiman Han}

The Affiliated Hospital of Qingdao University https://orcid.org/0000-0002-9230-5746

\section{Chuantao Zhang}

The Affiliated Hospital of Qingdao University

\section{Man Jiang}

The Affiliated Hospital of Qingdao University

\section{Tianjun Li}

The Affiliated Hospital of Qingdao University

\section{Jingjuan Zhu}

The Affiliated Hospital of Qingdao University

\section{$\mathrm{Na}$ Zhou}

The Affiliated Hospital of Qingdao University

\section{Helei Hou}

The Affiliated Hospital of Qingdao University

Xiaochun Zhang ( $\nabla$ zxc9670@qdu.edu.cn)

The Affiliated Hospital of Qingdao University https://orcid.org/0000-0001-5691-9946

\section{Research article}

Keywords: Adenocarcinoma, EGFR, ALK, Double mutation, Targeted therapy

Posted Date: December 28th, 2020

DOI: https://doi.org/10.21203/rs.3.rs-130711/v1

License: (1) This work is licensed under a Creative Commons Attribution 4.0 International License. Read Full License 


\section{Abstract}

Background: Epidermal growth factor receptor (EGFR) mutations and anaplastic lymphoma kinase (ALK) rearrangements are two driver alterations and are generally considered mutually exclusive in non-small cell lung cancer (NSCLC). The prevalence of EGFR/ALK co-alterations in patients with NSCLC is low, and the clinicopathological features and optimal targeted therapies of these subtype of patients are still controversial.

Methods: We describe three cases of NSCLC harboring EGFR mutation and ALK rearrangement. All of them received more-line therapies and showed the long-term survival benefit from targeted therapies.

In addition, we searched PUBMED, EMBASE and MEDLINE up to September, 2020. 91 EGFR/ALK coaltered patients of NSCLC included for analysis in our study. Survival curves were created by KaplanMeier method and group comparison analyses of progression free survival (PFS) were using log-rank test.

Result: A total of 91 patients were summarized in our study from previous literatures. The patients of NSCLC with coexisting EGFR mutations and ALK rearrangements are more likely to occur in female, nonsmoker, Asian origin, adenocarcinoma, and IV stage. The disease control rate (DCR) of tyrosine kinase inhibitors (TKIs) which targeted EGFR and ALK as first-line targeted therapy was $62 \%$ and $78 \%$, respectively. The median PFS on first EGFR-TKI and first ALK-TKI therapy were 5.3 months (95\% confidence interval $[\mathrm{Cl}] 1.20-9.40$ months) and 6.0 months (95\% $\mathrm{Cl} 0.00-14.69$ months) in EGFR/ALK co-altered NSCLC patients. Among patients who were treated with EGFR-TKI as first-line targeted therapy, univariant analysis showed that PFS have no significant difference between male and female $(p=0.22)$, and there is also no difference between Asian and Caucasian ( $p=0.939)$. The median PFS between firstand second-line targeted therapies was 7.0 months ( $95 \% \mathrm{Cl} 4.83-9.17$ months) and 2.0 months $(95 \% \mathrm{Cl}$ 0.96-3.05 months) $(p=0.075)$. Survival curves showed the significantly prolonged PFS between patients without and with CNS metastasis $(p=0.036)$.

Conclusion: Both EGFR-TKIs and ALK-TKIs have been proved their effectiveness to EGFR/ALK doublepositive NSCLC patients. The curative effect of combination targeted therapies and sequential treatment regimens are still in exploration.

\section{Introduction}

Lung cancer is the most common malignant tumor in the worldwide and remines the leading cause of cancer-related death, and non-small cell lung cancer (NSCLC) patients account for $80 \%-85 \%$ of all Lung cancer patients (1). It is transformed dramatically in the therapy regimens of NSCLC since the discovery of several critical activating pathways (2). In recent years, with the advent of targeted therapies for different oncogenic drivers, molecular analysis is routinely performed in NSCLC to seek for major therapeutic targets, especially in adenocarcinoma which accounts for approximately $40 \%$ of NSCLC. Epidermal growth factor receptor (EGFR) mutation occurring in almost $10-30 \%$ of the patients with 
NSCLC, which is the most common driving oncogene (3). The reported incidence of anaplastic lymphoma kinase (ALK) rearrangements ranges from 3-5\% in NSCLC patients and echinoderm microtubuleassociated proteinlike4 (EML4-ALK) accounts for more than $90 \%$ of ALK-rearrangement (4). Generally, these two driver genes are considered mutually exclusive in $\operatorname{NSCLC(5),~and~we~have~little~know~about~}$ clinicopathologic feature and treatment. There are several studies and case reports have reported the coexistence of EGFR mutations and ALK rearrangements(6-33), both EGFR TKIs and ALK TKIs show their therapeutic effects. Patients of NSCLC with EGFR/ALK double-positive show different sensitivities to various therapeutic strategies. But there is no general consensus on the optimal target therapy for EGFR/ALK double-positive patients.

The central nervous system (CNS) metastasis, including brain metastasis (BM) and Leptomeningeal metastasis (LM), occurs in 30-50\% of advanced NSCLC patients and associated with poor prognosis(34, 35). Cumulative reports have showed that the third-generation EGFR tyrosine kinase inhibitors (TKIs) as well as second- and third-generation ALK TKIs have intracranial activity(36-39), but their response to NSCLC with LM is conflicting.

Herein, we present a case series of 3 NSCLC patients with double EGFR/ALK positive that all received targeted therapies. In addition, we summarized 91 patients with this type of genotype, and analyzed their clinicopathological characteristics and response to targeted therapies. Our study aimed to access the effectiveness of targeted therapies in EGFR/ALK double-positive patients especially those with CNS metastasis.

\section{Case Description}

\section{Case 1}

A 45-year-old male, asymptomatic, heavy smoker (10 cigarettes a day) had an incidental finding of a lung occupying lesion from a computed tomography (CT) scan performed for health examination in March 2018. The chest CT revealed a mass which was $23 \mathrm{~mm}$ in maximum diameter in his superior lobe of right lung and enlargement of lymph nodules of the mediastinum and right hilar. He underwent right upper lobectomy with systematic nodal dissection. Acinar adenocarcinoma and papillary adenocarcinoma were confirmed by the pathological examination, and the right paratracheal and subcarinal lymph nodules were invaded by tumor ( $\mathrm{pT} 1 \mathrm{bN2M0}$, stage IIA). Next-generation sequencing (NGS) test for a large panel showed a deletion of exon 19 of EGFR gene and EML4-ALK rearrangement and the mutation abundance was $1.97 \%$ and $13.79 \%$. Subsequently, EGFR exon 19 confirmed by immunohistochemistry (IHC) and ALK rearrangement verified by fluorescence in situ hybridization (FISH). The patient Eastern Cooperation Oncology Group performance score (ECOG-PS) was 1 at one month after surgery, and he initially received adjuvant chemotherapy regimen with pemetrexed and nedaplatin (Day 1: $500 \mathrm{mg} / \mathrm{m}^{2}+$ Day 1-2: $80 \mathrm{mg} / \mathrm{m}^{2}, 21$ days/ cycle) in May 2018. After 6 cycles of chemotherapy, icotinib, a first generation of EGFR-TKI, (125 mg thrice daily) was selected as maintenance regimen. In December 2018, there was no inducement for the patient and he suffered headache, dizziness, nausea, projectile vomited and 
developed forced right lateral decubitus position. He had a positive Babinski sign and couldn't complete both hands alternating movement test and finger-nose test. Brain magnetic resonance imaging (MRI) revealed cerebellar metastasis (CM) and leptomeningeal carcinomatosis (LMC) (Fig. 1). The patient began treatment with ALK-TKI alectinib (600 mg twice daily) combined with icotinib (125 mg thrice daily). In the same time, mannitol was used to decrease intracranial pressure. Three days after treatment, the patient was able to walk, and headache, dizziness, nausea as well as vomit was relieved. Brain MRI scanning demonstrated a marked regression of LMC after 2 months. However, the therapy was ceased after 3 months because the patient suffered from dizziness, and brain MRI revealed leptomeningeal progression compared with before. Considering that alectinib was beneficial for brain metastasis, he then switched to take alectinib (600 mg twice daily) combined with Osimertinib (160 mg once daily). A month later, this therapy was terminated after further $\mathrm{CM}$ progression showed in brain MRI and three new lesions in cerebellum was confirmed. A pulmonary nodule, meanwhile, was revealed by chest CT scanning which was in left upper lobe. We tried to analyze circulating tumor cells and cell-free tumor DNA in cerebrospinal fluid (CSF), but no valuable information found. Subsequently, he began treated both brigatinib (180 mg once daily with 7-day lead-in period at $90 \mathrm{mg}$ ) and bevacizumab (Day 1:10 mg/kg, 21 days/ cycle) from June 2019. After one cycle of this therapy, brain MRI scanning and chest CT scanning revealed a decrease of brain metastasis and pulmonary metastasis. Nevertheless, brigatinib was discontinued after 6 months due to nausea, vomiting and loss of appetite. The patient was subsequently switched to lorlatinib (25 mg once daily) combined with bevacizumab (7.5 mg/kg D1, 21 days/ cycle), and lorlatinib followed was gradually incremented to $75 \mathrm{mg}$ daily. Mild tachycardia and hallucinations were apparent temporarily and recovered without any management. Brain MRI scan and chest CT scan were performed bimonthly that showed stable disease in the next 8 months of this treatment, and the patient remains on this therapy util reporting this case.

\section{Case 2}

A 48-year-old male non-smoker suddenly appear to be having an epileptic seizure and fell down in April 2018. Brian MRI revealed brain nodules and metastases cannot be rule out. FDG-PET scan was performed next, revealing the presence of a lesion in the lower right lung and multiple brain lesions. The pathological examination of CT guided percutaneous lung puncture biopsy revealed an adenocarcinoma, and we made a diagnosis of right lower lobe adenocarcinoma at stage IV. The patient had a good performance status and he was initially received first-line chemotherapy regimen of PP (pemetrexed $500 \mathrm{mg} / \mathrm{m}^{2}$ D1 + nedaplatin mg/ m² D1, 21 days a cycle) in May 2018 every three weeks up to 4 cycles. The patient had a stable disease (SD) and was performed NGS detection which identified L858Q mutation $(0.30 \%)$ in exon 21 concomitant with ALK-SH3RF3 fusion gene $(0.33 \%)$ in the tumor cell. Then, based on the molecular finding, he started to take first-generation ALK-TKI crozitinib (250 mg twice daily), and disease stability was achieved for 8 months (Fig. 2). At progression, he received EGFR-TKI gefitinib (250 mg once daily), but the drug was stopped for skin toxicity after 1 month. In April 2019, whole-brain radiation (WBRT) was given for 20 times. In June 2019, the patient started taking brigatinib (180 mg once daily with 7-day lead-in period at $90 \mathrm{mg}$ ) combined with icotinib (250 mg thrice daily). 5 months later, CT 
scans evidenced a partial response in the brain lesions. However, the progressive disease (PD) was confirmed by brain MRI in May 2020, with a progression-free survival (PFS) of 11 months. The therapy regimen was switched to alectinib (600 mg twice daily) combined with icotinib ( $250 \mathrm{mg}$ thrice daily). Unfortunately, the therapy response was assessed as PD with the efficacy evolution showed an enlarged tumor size after 2 months of this therapy. his cancer progressed again and he began taking ceritinib (750 mg Daily) combine with icotinib for about 1 month and then experienced PD. Next, the patient was treated with loratinib (100 mg daily) and the clinical response was not available util reporting this case.

\section{Case 3}

In March 2015, a 61-year-old female non-smoker who was asymptomatic was found a lung mass in the right lower lobe with malignant pleural effusion in physical examination. Percutaneous pulmonary biopsy was diagnosed of right lower lobe adenocarcinoma at stage IV, and the tumor tissue was too small to perform the gene test. The patient worried about the side effect of chemotherapy. In April 2015, she started to take gefitinib (250 mg once a day) and had a partial response after 3 months (mutation status was unknown) (Fig. 3). In August 2016, she began receiving temozolomide combined with gefitinib because of the discovery of brain lesions by MRI. In November 2016, pleural effusion was suddenly increased and considered the progression disease. Then, plasma-based NGS was performed and the EGFR mutation (T790M, exon20) was found. He was treated with AZD9291 since November 2016 and the disease stability was achieved for 11 months. In October 2017, CT-scan revealed the progression of pulmonary nodules as well as pleural effusion, and the patient was treated with CT-guided seed implantation and AZD9291 treatment was continued simultaneously. Then, a new lung biopsy specimen was obtained and NGS was performed, which showed the concomitant of EGFR exon 19 deletion and EML4-ALK rearrangement and the mutation abundance was $16.69 \%$ and $22.29 \%$, respectively. For this reason, the treatment regimen was switched to crozitinib ( $250 \mathrm{mg}$ twice daily) combined with AZD 9291 (80 mg daily) in January 2018. After 7 months of this therapy, the drug was discontinued for the presence of chest distress and breath obstruction. Followed CT scan revealed the massive right-side pleural effusion and left-sided pneumonia, and disease progression again was confirmed. We conducted rebiopsy and NGS to the patient, and the result of NGS was consist with the previous one. A right thoracic drainage and cis-platinum intrapleural infusion was performed in July 2018. Then, the patient initially received chemotherapy of PP (pemetrexed $500 \mathrm{mg} / \mathrm{m}^{2} \mathrm{D} 1$ + nedaplatin $\mathrm{mg} / \mathrm{m}^{2} \mathrm{D} 1,21$ days/cycle) plus bevacizumab (7.5 mg/kg D1, 21 days/cycle) and continued taking AZD9291. However, cardiac insufficient was happened to her on the fourth day of 7th cycle chemotherapy with the left ventricular ejection fraction of $44 \%$. Thus, she ceased to take AZD9291 and continued the pemetrexed (pemetrexed $500 \mathrm{mg} / \mathrm{m}^{2}$ D1, 21 days/cycle) plus bevacizumab (7.5 mg/kg D1, 21 days/cycle) for another 22 cycles and stable disease was achieved. In April 2020, the patient suffered with headache and dizziness with an unsteady step, and LM) couldn't be rule out but we were not obtained evidence from imaging test. From May 2020 to reporting this case (September 2020), the patient was treated with Osimertinib (160 mg, once a day). After 4 months, CT scan showed a right-side pleural effusion was increased again with elevated levels of carcinoma embryonic antigen (CEA), and the Osimertinib was discontinued. 


\section{Materials And Methods}

We searched PUBMED, EMBASE and MEDLINE for the patients of NSCLC harboring both EGFR mutation and ALK rearrangement. With this methodology, we found 24 literatures and total of 91 patients were fulfilled these criteria (10-33). Clinical data including gender, age, ethnicity, smoking history, pathological, tumor staging, CNS metastasis and the efficacy of targeted therapies. The PFS was the primary endpoint to assess the efficacy of TKIs, and overall survival (OS) and DCR was the secondary outcomes. Statistical Package for Social Science version 23.0 was used to perform statistical analysis. Survival curves were created by Kaplan-Meier method and group comparison analyses of PFS were using log-rank test. We defined statistically significant was $p$ value $<0.05$.

\section{Result}

Table 1 summarizes detailed characteristics of 91 EGFR/ALK double-positive NSCLC patients which described by published data. The baseline demographic and clinical characteristics was summarized in Table 2, including age, gender, ethnicity, smoking history, pathology, tumor stage, EGFR mutation types and CNS metastases. Among these people, the clinical information of 88 patients was described in details. The median age of these patients was 59,4 years. $96.6 \%(85 / 88)$ of them were adenocarcinoma, and they were more likely to occur in female, non-smoker, Asian origin, IV stage. Of all co-mutation patients, 19 exon (-del) and 21 exon (-L858R) were frequently-occurring EGFR gene mutations, and CNS metastasis were proved exist in 11 patients.

Most EGFR/ALK co-alteration NSCLC patients (78/90) were treated with at least one kind of TKIs. Because of some patients not be able to get complete data, and only 53 patients could follow the evaluating clinical effect. Among these patients, 23 of them only received EGFR TKIs, 12 patients only received ALK TKIs, and 45 patients received combination or sequential therapy of both EGFR-TKI and ALK-TKI. 29 out 53 patients were receiving EGFR TKIs as first-line targeted therapy and other 24 patients choose ALK TKIs with a DCR of $62 \%$ and $78 \%$, respectively.

The median PFS on first EGFR-TKI and first ALK-TKI therapy were 5.3 months ( $95 \%$ confidence interval [Cl] 1.20-9.40 months) and 6.0 months (95\% $\mathrm{Cl} 0.00-14.69$ months) in EGFR/ALK co-altered NSCLC patients. Univariant analysis showed that PFS have no significant difference $(p=0.132)$ (Fig. 4a.). Among patients who were treated with EGFR-TKI as first-line targeted therapy, the median PFS was 7.0 months (95\% $\mathrm{Cl} 0.75-13.25$ months) and 7.7 months (95\% Cl 4.08-11.32 months) in male and female (Fig. 4b.), and was 6.0 months ( $95 \% \mathrm{Cl} 2.91-9.10$ months) and 7.0 months ( $95 \% \mathrm{Cl} 0.53-13.48$ months) in Asian and Caucasian (Fig. 4c.). We can draw a conclusion that there was no statistically significant difference between male and female $(p=0.22)$ as well as Asian and Caucasian $(p=0.939)$. Additionally, the median PFS between first- and second-line targeted therapies was 7.0 months (95\% Cl 4.83-9.17 months) and 2.0 months ( $95 \% \mathrm{Cl}$ 0.96-3.05 months), which showed no statistically different between these two groups $(p=0.075)$ (Fig. 4d.). Survival curves showed the significantly prolonged PFS between patients without and with CNS metastases $(p=0.036)$, which median PFS was 8.0 month $(95 \% \mathrm{Cl} 0.53-13.48$ 
months) and 2.0 month ( $95 \% \mathrm{Cl} 0.53-13.48$ month) (Fig. 4e). Only 14 patients can get the data of OS while the other missing data were not available, the average OS was 56.18 months $(95 \% \mathrm{Cl} 32.72-72.9$ months) (Fig. 4f).

\section{Discussion}

EGFR/ALK double-positive is a relatively rare molecular subtype in NSCLC, which accounts for 0.1 to $1.6 \%$ in NSCLC according to the former studies $(9,40-43)$, and present in $3.9 \%$ and $18.6 \%$ of patients with EGFR mutation and ALK rearrangement respectively(29). The prevalence of concomitant EGFR mutations and ALK alterations correlates with ethnic differences as well as detection sensitivity. Along with the discovery of more sensitive gene detection technologies, such as NGS, the proportion of double-positive patients may much higher and treatment strategies for NCSLC patients have a remarkable evolution. EGFR mutation usually occurs in exon 19 deletion (60\%) and L858R missense substitutions in exon 21 (35\%). L861Q point mutation in exon21 accounts for only $2 \%$ of EGFR mutation(44).

NSCLC patients harboring driver gene mutations such as EGFR mutations and ALK rearrangements could benefit more from molecular targeted therapies than conventional chemotherapies(45). Now, EGFR- and ALK-TKI are standard therapies for NSCLC patients with single gene alterations. Previous studies had revealed the efficacy of EGFR- and ALK-TKI in NSCLC patients with EGFR/ALK dule-positive, but the sensitivity of these TKIs remains controversial. Based on previous research, EGFR mutations were considered one of the important resistance mechanisms of ALK-TKI(28, 46), and EGFR-TKI combined with ALK-TKI seems to receive more benefit. While Koivunen et al. reported that ALK rearrangement is a potential resistance mechanism to EGFR-TKI(47). Yang JJ et al. assessed the response of double-positive patients to EGFR TKIs and first-generation ALK-TKI crozitinib. He came to a conclusion that the levels of phospho-EGFR and phosphor-ALK could seem as the prognosis index of EGFR-TKI and crozitinib in NSCLC patients who overlapping EGFR mutations and ALK rearrangement (29). Won et al. reported that double-positive patients showed gefitinib resistance but were sensitive to ALK-TKI(30), suggesting that ALK-TKI can be selected firstly in double-positive patients. Nevertheless, Schmid et al. found that sensitivity to EGFR TKIs was higher than ALK TKIs in patients with EGFR/ALK co-alterations(48). Furthermore, Zhao et al. reported that the OS of concomitant patients appears longer than those with single EGFR mutation(43), while Luo et al. put forward to contrary option (40).

Here, we illustrate 3 NSCLC patients harboring EGFR mutation and ALK rearrangement who received more-line targeted therapies. Patients 1 intracranial responded quickly to alectinib with neurological symptoms recovered satisfactorily. The second-generation ALK-TKI alectinib showed clinically meaningful in CNS metastasis ALK-positive NSCLC with a highly CNS-penetrant(49, 50). In patients of NSCLC harboring EGFR-sensitive mutations, it was confirmed that EGFR TKIs combined with humanized monoclonal antibody targeting vascular endothelial growth factor (VEGF) bevacizumab can improve the antitumor effect compared to EGFR therapy alone(51-53). Dule inhibition of both EGFR and VEGF pathway could treat or delay the resistance to EGFR inhibitors(51). He received third-generation ALK-TKI lorlatinib combined bevacizumab after failing on 3 previous targeted therapies and achieved SD for more 
than 8 months until the last follow-up time (September 2020). Patient 2 had a PR to second-line brigatinib /icotinib combination therapy after first-line chemotherapy followed by icotinib as maintenance therapy. However, he was primary refractory to third-line and fourth-line dual EGFR/ALK combination therapies and received loratinib as fifth-line therapy. Patient 3 had massive system and intracranial progression, who had a PR to first-line gefitinib and second-line AZD9291. And she had a SD to fifth-line therapy of third-generation EGFR-TKI osimertinib which showed ongoing tumor response. Until last follow-up, the OS of our three cases was 29 months, 28 months, and 53 months.

To data, the clinicopathological features of EGFR/ALK co-altered patients were not been accurately described in further studies. Cai et al.(8) proposed that EGFR/ALK double gene co-expressed due to intratumor heterogeneity. While Sasaki et al(54) found that coexistence of EGFR signaling could occurs in an ALK-rearrangement mutation cell line. We aim to help determine the clinicopathological features and targeted therapeutic agent for NSCLC patients with EGFR/ALK double-positive and collect 91 cases were identified in this double-positive by September 2020 in previous publications. In our study no significant correlation in terms of response to targeted therapies in gender as well as ethnicity. Limited data were available in the previous literatures. Luo et. al (40) reported that patients of NSCLC with EGFR/ALK double-positive appears to be associated with shorter median OS compared with single EGFR mutation and ALK rearrangement, and the median OS of was 18.5 months, 21.3 months and 23.7 months, respectively $(p=0.06)$. Zhao et al. (55) reported that sequential treatment with EGFR- and ALK-TKIs were effective in treatment of double-positive patients, and these patients toward increased long-term survival compared with single positive of EGFR and ALK. Since of the limited numbers of patients can acquired the data that we cannot draw firm conclusions to evaluate overall survival of these EGFR/ALK co-altered patients. Moreover, chemotherapy and radiation therapy might be interference factors with the results.

In our study, univariate analysis showed that PFS in non-CNS metastases subgroup was superior to that CNS metastases. CNS metastases occurs in $22 \%-44 \%$ of patients with advance NSCLC, and it often associated with poor prognosis and quality of life in advance NSCLC patients(56). The median survival of NSCLC patients with BM and LM was 7 month and 3-10 months $(57,58)$, respectively. Patients of NSCLC with targetable mutation such as EGFR mutation and ALK rearrangement seems to have higher incidence of CNS metastases(59-61). LM occurs in approximately 3\%-4\% of patients with advanced NSCLC and 9\% in EGFR-mutant advanced NSCLC $(58,62,63)$. Some EGFR- and ALK-TKIs such as osimertinib, alectinib, brigatinib, ceritinib, lorlatinib have demonstrated their intracranial activity. All of our three patients suffered from CNS metastasis, one of them have LM and another one suspected LM. It is less known about the efficacy of EGFR TKIs and ALK TKIs in patients with LM since that the patients are often excluded from clinical trials. There are several case reports revealed the efficacy of the second-and third-generation of ALK-TKI on LM in NSCLC(64-66).

The third-generation EGFR-TKI osimertinib has the high CNS penetration with higher cerebrospinal fluid (CSF) distribution compared with other EGFR TKIs(67-69), and NCCN has recommend osimertinib to treat EGFR-mutant NSCLC patients with CNS metastases(70). Based on the phase III ALEX study, alectinib shows an excellent efficacy in ALK-rearrangement NSCLC including CNS metastases (30). A study by 
Gainor JF et al. demonstrated the efficacy of alectinib in ALK-rearrangement NSCLC with LM (71). Brigatinib, a second-generation ALK TKI, exhibits the efficacy in osimertinib resistance of EGFR C797S alteration(72). A meta-analysis shows that brigatinib may have some advantages over alectinib in CNS metastasis, but it requires further validation(73). ALK-TKIs have demonstrated their clinical success in tumors with activation of ALK but eventually limited by the development of drug resistance. The mechanism of medicine resistance to TKIs may mutual independence in double-positive patient population. The third-generation ALK-TKI lorlatinib was approved by the United State food and drug administration (FDA) for the treatment of advance NCLC after progression on first- and second-generation ALK TKIs including alectinib and ceritinib or after crozitinib and at least one other ALK-TKI(74). It has excellent CNS penetration and efficacy in CNS metastasis after disease progression on other first- and second-generation ALK TKIs(75-77). We reported three patients of NSCLC with EGFR/ALK dual-altered with LM who showed intra-cranial response to the third-generation EGFR-TKI and second- and thirdgeneration ALK-TKIs.

\section{Conclusion}

Here, we describe 3 cases and made a retrospective study with a relatively small sample because of the low incidence of EGFR and ALK dual mutations. There are some limitations in our study since that we can hardly acquire the large sample of this kind of genotype to analyze the biological behavior, clinicopathologic features and the optimal therapeutic regimens. The implementation of sensitive molecular genetic technology, such as NGS, led to the increase in frequency of these patients. The researches of resistance mechanisms and sequential treatment regimens in EGFR/ALK double-positive are little. Sasaki et al. found that EGFR/ALK double positive cancer cell line was expected to be resistant to both EGFR- and ALK-TKIs(28). In our 3 cases, combination targeted therapies showed good prospect to double-altered patients. It requires more researches to explore the optimal treatment regimen for this dualaltered NSCLC in the future.

\section{Abbreviations}

\section{EGFR}

Epidermal growth factor receptor; ALK:Anaplastic lymphoma kinase; NSCLC:Non-small cell lung cancer; DCR:Disease control rate; TKI:Tyrosine kinase inhibitor; WBRT:Whole-brain radiation; PD:Progressive disease; PFS:Progression free survival; Cl:Confidence interval ; CNS:Central nervous system ; LM:Leptomeningeal metastasis; CT:Computed tomography; NGS:Next-generation sequencing; IHC:Immunohistochemistry ; FISH:Fluorescence in situ hybridization; ECOG-PS:Eastern Cooperation Oncology Group performance score; MRI:magnetic resonance imaging; CM:cerebellar metastasis; LMC:Ieptomeningeal carcinomatosis; CSF:cerebrospinal fluid; CEA:carcinoma embryonic antigen ; OS:overall survival ; FDA:food and drug administration

\section{Declarations}


Acknowledgements

We thank the reviewer for their constructive comments.

\section{Authors contribution}

Xiaochun Zhang conceived the study, and performed the analysis. Qiman Han and Man Jiang summarized the data, and designed the figures. $\mathrm{Na}$ Zhou and Chuantao Zhang analyzed and interpreted the data. All authors read and approved the final version of the manuscript.

\section{Funding}

There is no funding to report.

\section{Availability of data and materials}

The data that support the findings of this study are available, upon reasonable request.

\section{Ethics approval and consent to participate}

This study was approved by the Ethics Committee of the Affiliated Hospital of Qingdao University. Written informed consent was obtained from the patients included in the study.

\section{Consent for publication}

Not applicable.

\section{Competing interests}

The authors declare that they have no competing interests.

\section{Authors details}

${ }^{1}$ Center for Precision Center Medicine, the Affiliated Hospital of Qingdao University, Qingdao 266000, Qingdao, China.

\section{References}

1. Siegel RL, Miller KD, Jemal A. Cancer statistics. 2018. CA: A Cancer Journal for Clinicians. 2018;68(1):7-30.

2. Kris MG, Johnson BE, Berry LD, Kwiatkowski DJ, lafrate AJ, Wistuba II, et al. Using multiplexed assays of oncogenic drivers in lung cancers to select targeted drugs. JAMA. 2014;311(19):19982006.

3. Hirsch FR, Bunn PA. EGFR testing in lung cancer is ready for prime time. Lancet Oncology. 2009;10(5):432-3. 
4. Soda M, Choi YL, Enomoto M, Takada S, Yamashita Y, Ishikawa S, et al. Identification of the transforming EML4-ALK fusion gene in non-small-cell lung cancer. Nature. 2007;448(7153):561-6.

5. Gainor JF, Varghese AM, Ou SH, Kabraji S, Awad MM, Katayama R, et al. ALK rearrangements are mutually exclusive with mutations in EGFR or KRAS: an analysis of 1,683 patients with non-small cell lung cancer. Clinical cancer research: an official journal of the American Association for Cancer Research. 2013;19(15):4273-81.

6. Lung cancers with. concomitant EGFR mutations and ALK rearrangements: diverse responses to EGFR-TKI and crizotinib in relation to diverse receptors phosphorylation. Clinical Cancer Research An Official Journal of the American Association for Cancer Research. 2014;20(5):1383.

7. Russo GL, Imbimbo M, Corrao G, Proto C, Garassino MC. Concomitant EML4-ALK rearrangement and EGFR mutation in non small cell lung cancer patients: A literature review of 100 cases. Oncotarget. 2017;8(35).

8. Cai W, Lin D, Wu C, Li X, Zhao C, Zheng L, et al. Intratumoral Heterogeneity of ALK-Rearranged and ALK/EGFR Coaltered Lung Adenocarcinoma. Journal of Clinical Oncology. 2015:3701-9.

9. Won JK, Keam B. J., et al. Concomitant ALK translocation and EGFR mutation in lung cancer: a comparison of direct sequencing and sensitive assays and the impact on responsiveness to tyrosine kinase inhibitor. Annals of Oncology. 2014.

10. Lee JK, Kim TM, Koh Y, Lee SH, Kim DW, Jeon YK, et al. Differential sensitivities to tyrosine kinase inhibitors in NSCLC harboring EGFR mutation and ALK translocation. Lung Cancer. 2012;77(2):4603.

11. Tiseo M, Gelsomino F, Boggiani D, Bortesi B, Bartolotti M, Bozzetti C, et al. EGFR and EML4-ALK gene mutations in NSCLC: A case report of erlotinib-resistant patient with both concomitant mutations. Lung Cancer. 2011;71(2):241-3.

12. Chen X, Zhang J, Hu Q, Li X, Zhou C. A case of lung adenocarcinoma harboring exon 19 EGFR deletion and EML4-ALK fusion gene. Lung Cancer. 2013;81(2):308-10.

13. Tanaka H, Hayashi A, Morimoto T, Taima K, Tanaka Y, Shimada M, et al. A case of lung adenocarcinoma harboring EGFR mutation and EML4-ALK fusion gene. Bmc Cancer. 2012;12(1).

14. Baldi L, Mengoli MC, Bisagni A, Banzi MC, Boni C, Rossi G. Concomitant EGFR mutation and ALK rearrangement in lung adenocarcinoma is more frequent than expected: Report of a case and review of the literature with demonstration of genes alteration into the same tumor cells. Lung Cancer. 2014;86(2):291-5.

15. Chiari R, Duranti S, Ludovini V, Bellezza G, Pireddu A, Minotti V, et al. Long-term response to gefitinib and crizotinib in lung adenocarcinoma harboring both epidermal growth factor receptor mutation and EML4-ALK fusion gene. J Clin Oncol. 2014;32(9):30-2.

16. Kuo Y-W, Wu S-G, Ho C-C, Shih J-Y. Good response to gefitinib in lung adenocarcinoma harboring coexisting EML4-ALK fusion gene and EGFR mutation. 2010.

17. Miyanaga A, Shimizu K, Noro R, Seike M, Gemma A. Activity of EGFR-tyrosine kinase and ALK inhibitors for EML4-ALK -rearranged non-small-cell lung cancer harbored coexisting EGFR 
mutation. Bmc Cancer. 2013;13(1):262-.

18. Zhao N, Zheng SY, Yang JJ, Zhang XC, Xie Z, Xie B, et al. Lung Adenocarcinoma Harboring Concomitant EGFR Mutation and EML4-ALK Fusion That Benefits From Three Kinds of Tyrosine Kinase Inhibitors: A Case Report and Literature Review. Clinical Lung Cancer. 2015;16(2):e5-9.

19. Zhou J, Jing Z, Jing Z, Sheng Y, Zhou J. Poor response to gefitinib in lung adenocarcinoma with concomitant EGFR mutation and ALK rearrangement. Thoracic Cancer. 2014;6(2).

20. Fan T, Song YJ, Liu XL. Adenocarcinoma of the lung with concomitant ALK fusion gene and EGFR gene mutation: A case report and literature review. Mol Clin Oncol. 2016;4(2):203-5.

21. Popat S, Vieira de Araujo A, Min T, et al. Lung adenocarcinoma with concurrent exon 19 EGFR mutation and ALK rearrangement responding to erlotinib. J Thorac Oncol. 2011;6(11).

22. Santelmo C, Ravaioli A, Barzotti E, et al. Coexistence of EGFR mutation and ALK translocation in NSCLC. Lung Cancer. 2013;82(2):294-6.

23. Thumallapally N, Yu H, Farhan M, Ibrahim U, Odiami M. Concomitant Presence of EGFR and ALK Fusion Gene Mutation in Adenocarcinoma of Lung: A Case Report and Review of the Literature. Journal of Pharmacy Practice. 2018;31(3):897190017704751.

24. Caliez J, Monnet I, Pujals A, Rousseaubussac G, Jabot L, Boudjemaa A, et al. [Lung adenocarcinoma with concomitant EGFR mutation and ALK rearrangement]. Revue Des Maladies Respiratoires. 2016;34(5):576.

25. Sweis RF, Thomas S, Bank B, Fishkin P, Salgia R. Concurrent EGFR Mutation and ALK Translocation in Non-Small Cell Lung Cancer. Cureus. 2016;8(2):e513.

26. Schmid S, Gautschi O, Rothschild S, Mark M, Froesch P, Klingbiel D, et al. Clinical Outcome of ALK Positive Non-Small Cell Lung Cancer (NSCLC) Patients with de novo EGFR or KRAS Co-Mutations Receiving Tyrosine Kinase Inhibitors (TKI). Journal of Thoracic Oncology. 2017;12(4):681-8.

27. Ulivi P, Chiadini E, Dazzi C, Dubini A, Delmonte A. Nonsquamous. Non-Small-Cell Lung Cancer Patients Who Carry a Double Mutation of EGFR, EML4-ALK or KRAS: Frequency, Clinical-Pathological Characteristics, and Response to Therapy. Clinical Lung Cancer. 2016;17(5):384-90.

28. Sasaki T, Koivunen J, Ogino A, Yanagita M, Nikiforow S, Zheng W, et al. A novel ALK secondary mutation and EGFR signaling cause resistance to ALK kinase inhibitors. Cancer Res. 2011;71(18):6051-60.

29. Yang JJ, Zhang XC, Su J, Xu CR, Zhou Q, Tian HX, et al. Lung Cancers with Concomitant EGFR Mutations and ALK Rearrangements: Diverse Responses to EGFR-TKI and Crizotinib in Relation to Diverse Receptors Phosphorylation. Clinical Cancer Research. 2014.

30. Won JK, Keam B, Koh J, Cho HJ, Jeon YK, Kim TM, et al. Concomitant ALK translocation and EGFR mutation in lung cancer: a comparison of direct sequencing and sensitive assays and the impact on responsiveness to tyrosine kinase inhibitor. Ann Oncol. 2015;26(2):348-54.

31. Shin HJ, Kho BG, Kim MS, Park HY, Oh IJ. Co-alteration of EGFR mutation and ALK rearrangement in non-small cell lung cancer: Case series. Medicine. 2019;98(9):e14699. 
32. Jing Z, Ming, Zou, Jinyan Y, et al. Effective treatment of pulmonary adenocarcinoma harboring triple EGFR mutations of L858R, T790M, and cis-C797S by osimertinib, bevacizumab, and brigatinib combination therapy: a case report. OncoTargets therapy. 2018;11:5545-50.

33. Akira Y, Atsuhisa T, Kazuko M, Kei K, Masahiro S, Takashi H, et al. Pulmonary Adenocarcinoma, Harboring Both an EGFR Mutation and ALK Rearrangement, Presenting a Stable Disease to Erlotinib and a Partial Response to Alectinib: A Case Report. Internal Medicine. 2018.

34. Remon J, Le Rhun E, Besse B. Leptomeningeal carcinomatosis in non-small cell lung cancer patients: A continuing challenge in the personalized treatment era. Cancer Treat Rev. 2017;53:128-37.

35. Staging Lung Cancer. Metastasis Radiol Clin North Am. 2018;56(3):411-8.

36. Ramalingam SS, Vansteenkiste J, Planchard D, Cho BC, Gray JE, Ohe Y, et al. Overall Survival with Osimertinib in Untreated, EGFR-Mutated Advanced NSCLC. The New England Journal of Medicine. 2020;382(1):41-50.

37. Rafal Dziadziuszko ADRC, Solange Peters B, D TM C, E JN. E MN, et al. Updated Efficacy and Safety Data and Impact of the EML4-ALK Fusion Variant on the Efficacy of AlectinibinUntreated ALK Positive Advanced Non-Small CellLung Cancer in the Global Phase III ALEX Study. Journal of Thoracic Oncology. 2019;14(7):1233-43.

38. Gaye E, Geier M, Bore P, Guilloïque M, Lucia F, Quéré G, et al. Intra-cranial efficacy of brigatinib in an ALK-positive non-small cell lung cancer patient presenting leptomeningeal carcinomatosis. Lung Cancer. 2019.

39. Solomon BJ, Besse B, Bauer TM, Felip E, Soo RA, Camidge DR, et al. Lorlatinib in patients with ALKpositive non-small-cell lung cancer: results from a global phase 2 study. The Lancet Oncology. 2018;19.

40. Lou NN, Zhang XC, Chen HJ, Zhou Q, Yang JJ. Clinical outcomes of advanced non-small-cell lung cancer patients with EGFR mutation, ALK rearrangement and EGFR / ALK co-alterations. Oncotarget. 2016;7(40):65185-95.

41. Lee JK, Kim TM, Koh Y, Lee S, Kim D, Jeon Y, et al. Differential sensitivities to tyrosine kinase inhibitors in NSCLC harboring EGFR mutation and ALK translocation. Lung Cancer. 2012;77(2):4603.

42. Ulivi P, Chiadini E, Dazzi C, Dubini A, Costantini M, Medri L, et al. Nonsquamous, Non-Small-Cell Lung Cancer Patients Who Carry a Double Mutation of EGFR, EML4-ALK or KRAS: Frequency, ClinicalPathological Characteristics, and Response to Therapy. Clinical Lung Cancer. 2016;17(5):384-90.

43. Zhao Y, Wang S, Zhang B, Qiao R, Xu J, Zhang L, et al. Clinical Management of Non-Small Cell Lung Cancer with Concomitant EGFR Mutations and ALK Rearrangements: Efficacy of EGFR Tyrosine Kinase Inhibitors and Crizotinib. Target Oncol. 2019;14(2):169-78.

44. Zhang X, Chang AY. Molecular predictors of EGFR-TKI sensitivity in advanced non-small cell lung cancer. Int J Med Sci. 2008;5(4):209-17.

45. Mitsudomi T, Morita S, Yatabe Y, Negoro S, Okamoto I, Tsurutani J, et al. Gefitinib versus cisplatin plus docetaxel in patients with non-small-cell lung cancer harbouring mutations of the epidermal 
growth factor receptor (WJTOG3405): an open label, randomised phase 3 trial. Lancet Oncology. 2010;11(2):121-8.

46. Kim SY, Kim TM, Kim D, Go H, Keam B, Lee S, et al. Heterogeneity of Genetic Changes Associated with Acquired Crizotinib Resistance in ALK-Rearranged Lung Cancer. Journal of Thoracic Oncology. 2013;8(4):415-22.

47. Koivunen J, Mermel CH, Zejnullahu K, Murphy C, Lifshits E, Holmes AJ, et al. EML4-ALK fusion gene and efficacy of an ALK kinase inhibitor in lung cancer. Clin Cancer Res. 2008;14(13):4275-83.

48. Schmid S, Gautschi O, Rothschild SI, Mark M, Froesch P, Klingbiel D, et al. Clinical Outcome of ALKPositive Non-Small Cell Lung Cancer (NSCLC) Patients with De Novo EGFR or KRAS Co-Mutations Receiving Tyrosine Kinase Inhibitors (TKIs). Journal of Thoracic Oncology. 2017;12(4):681-8.

49. Hida T, Nokihara $\mathrm{H}$, Kondo M, Kim YH, Tamura T. Alectinib versus crizotinib in patients with ALKpositive non-small-cell lung cancer (J-ALEX): an open-label, randomised phase 3 trial. Lancet. 2017;390(10089):29-39.

50. Peters S, Camidge DR, Shaw AT, Gadgeel S, Mok T. Alectinib versus Crizotinib in Untreated ALKPositive Non-Small-Cell Lung Cancer. N Engl J Med. 2017;377(9):829.

51. Combined vascular endothelial. growth factor receptor and epidermal growth factor receptor (EGFR) blockade inhibits tumor growth in xenograft models of EGFR inhibitor resistance. Clinical Cancer Research An Official Journal of the American Association for Cancer Research. 2009;15(10):348494.

52. Masuda C, Yanagisawa M, Yorozu K, Kurasawa M, Yamamoto K. Bevacizumab counteracts VEGFdependent resistance to erlotinib in an EGFR-mutated NSCLC xenograft model. Int J Oncol. 2017; $51(2): 425-34$.

53. Takashi S, Terufumi, Kato, Makoto N, et al. Erlotinib alone or with bevacizumab as first-line therapy in patients with advanced non-squamous non-small-cell lung cancer harbouring EGFR mutations (J025567): an open-label, randomised, multicentre, phase 2 study. Lancet Oncology. 2014.

54. Sasaki T, Koivunen J, Ogino A, Yanagita M, Nikiforow S, Zheng W, et al. A Novel ALK Secondary Mutation and EGFR Signaling Cause Resistance to ALK Kinase Inhibitors. Cancer Res. 2011;71(18):6051-60.

55. Yiming Z, Shuyuan, Wang, Bo Z, et al. Clinical Management of Non-Small Cell Lung Cancer with Concomitant EGFR Mutations and ALK Rearrangements: Efficacy of EGFR Tyrosine Kinase Inhibitors and Crizotinib. Targeted oncology. 2019.

56. Peters S, Bexelius C, Munk V, LeighI NB. The impact of brain metastasis on quality of life, resource utilization and survival in patients with non-small-cell lung cancer. Cancer Treat Rev. 2016;45:13962.

57. Xu Y, Hu M, Zhang M, Zhong W, Yin X, Sun Y, et al. Prospective study revealed prognostic significance of responses in leptomeningeal metastasis and clinical value of cerebrospinal fluid-based liquid biopsy. Lung Cancer. 2018;125:142-9. 
58. Li Y, Jiang B, Yang J, Tu H, Zhou Q, Guo W, et al. Leptomeningeal Metastases in Patients with NSCLC with EGFR Mutations. Journal of Thoracic Oncology. 2016;11(11):1962-9.

59. Zhang I, Zaorsky NG, Palmer JD, Mehra R, Lu B. Targeting brain metastases in ALK-rearranged nonsmall-cell lung cancer. Lancet Oncology. 2015;16(13).

60. Shin D, Na II, Kim CH, Park S, Baek H, Yang SH. EGFR Mutation and Brain Metastasis in Pulmonary Adenocarcinomas. Journal of Thoracic Oncology. 2014;9(2):195-9.

61. Shin DY, Na II, Kim CH, Park S, Baek HJ, Yang SH. EGFR Mutation and Brain Metastasis in Pulmonary Adenocarcinomas. Journal of thoracic oncology: official publication of the International Association for the Study of Lung Cancer. 2014;9(2):195-9.

62. Liao B, Lee J, Lin C, Chen Y, Chang C, Ho C, et al. Epidermal Growth Factor Receptor Tyrosine Kinase Inhibitors for Non-Small-Cell Lung Cancer Patients with Leptomeningeal Carcinomatosis. Journal of Thoracic Oncology. 2015;10(12):1754-61.

63. Kuiper JL, Hendriks L, Der Wekken AJV, De Langen AJ, Bahce I, Thunnissen E, et al. Treatment and survival of patients with EGFR-mutated non-small cell lung cancer and leptomeningeal metastasis: A retrospective cohort analysis. Lung Cancer. 2015;89(3):255-61.

64. Ou SI, Sommers KR, Azada MC, Garon EB. Alectinib Induces a Durable (> 15 Months) Complete Response in an ALK-Positive Non-Small Cell Lung Cancer Patient Who Progressed on Crizotinib With Diffuse Leptomeningeal Carcinomatosis. Oncologist. 2015;20(2):224-6.

65. Gaye E, Geier M, Bore P, Guilloique M, Lucia F, Quere G, et al. Intra-cranial efficacy of brigatinib in an ALK-positive non-small cell lung cancer patient presenting leptomeningeal carcinomatosis. Lung Cancer. 2019;133:1-3.

66. Gafer H, De Waard Q, Compter A, Den Heuvel MMV. Rapid regression of neurological symptoms in patients with metastasised ALK + lung cancer who are treated with lorlatinib: a report of two cases. Case Reports. 2019;12(7).

67. Yang JC, Kim S, Kim D, Lee JS, Cho BC, Ahn J, et al. Osimertinib in Patients With Epidermal Growth Factor Receptor Mutation-Positive Non-Small-Cell Lung Cancer and Leptomeningeal Metastases: The BLOOM Study. J Clin Oncol. 2020;38(6):538-47.

68. Ballard P, Yates JWT, Yang Z, Kim D, Yang JC, Cantarini M, et al. Preclinical Comparison of Osimertinib with Other EGFR-TKIs in EGFR-Mutant NSCLC Brain Metastases Models, and Early Evidence of Clinical Brain Metastases Activity. Clin Cancer Res. 2016;22(20):5130-40.

69. Colclough N, Ballard P, Barton P, Chen K, Cross D, Finlay MRV, et al. Preclinical comparison of the blood brain barrier (BBB) permeability of osimertinib (AZD9291) with other irreversible next generation EGFR TKIs. European Journal of Cancer. 2016;69.

70. National Comprehensive Cancer Network. 2019 [updated 2019. Available from: https://www.nccn.org/professionals/physician_gls/pdf/nsclpdf.

71. Justin F, Gainor, Carol A, Sherman, et al. Alectinib Salvages CNS Relapses in ALK-Positive Lung Cancer Patients Previously Treated with Crizotinib and Ceritinib. Journal of Thoracic Oncology. 2015. 
72. b AAML. AB, C EF, B RK. New therapeutic approaches to overcoming resistant EGFR exon 20 alterations. Critical Reviews in Oncology/Hematology.151.

73. Ando K, Akimoto K, Sato H, Manabe R, Kishino Y, Homma T, et al. Brigatinib and Alectinib for ALK Rearrangement-Positive Advanced Non-Small Cell Lung Cancer With or Without Central Nervous System Metastasis: A Systematic Review and Network Meta-Analysis. Cancers. 2020;12(4).

74. Yahiya S. Lorlatinib: First Global Approval. Drugs. 2019.

75. Zou HY, Friboulet L, Kodack DP, Engstrom LD, Li Q, West M, et al. PF-06463922, an ALK/ROS1 Inhibitor, Overcomes Resistance to First and Second Generation ALK Inhibitors in Preclinical Models. Cancer cell. 2015;28(1):70-81.

76. Bearz A, Gadgeel SM, Chiari R, Soo RA, Clancy JS, Seto T, et al. Lorlatinib in patients with ALKpositive non-small-cell lung cancer: results from a global phase 2 study. The lancet oncology. 2018;19(12):1654-67.

77. Gainor JF, Dardaei L, Yoda S, Friboulet L, Leshchiner I, Katayama R, et al. Molecular Mechanisms of Resistance to First- and Second-Generation ALK Inhibitors in ALK-Rearranged Lung Cancer. other. 2016;6(10).

\section{Tables}

Due to technical limitations, table 1,2,3 is only available as a download in the Supplemental Files section.

\section{Figures}



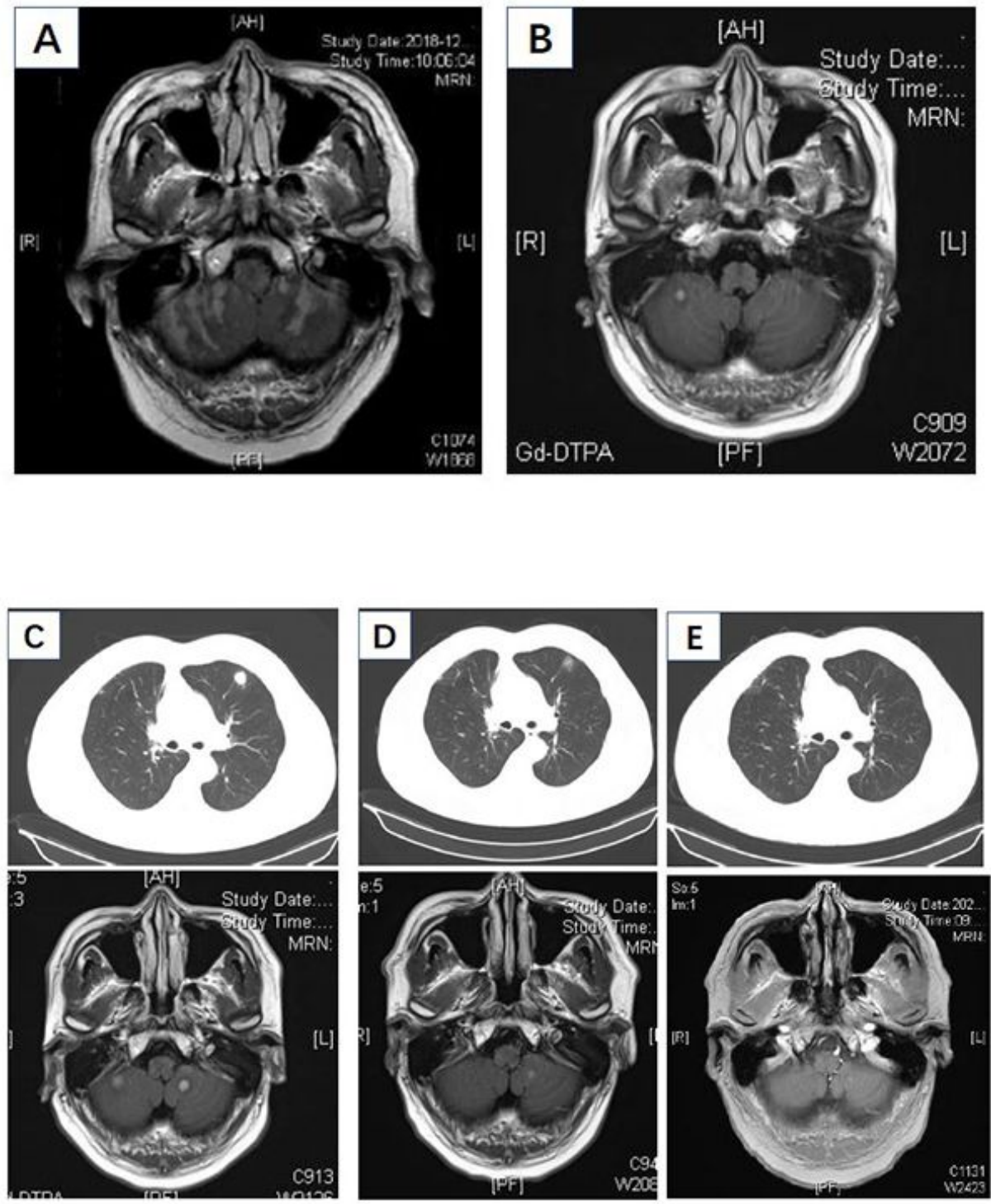

\section{Figure 1}

Serial MRI and CT of case 1. (A) 2018.12,before took TKIs. (B) 2019.3, alectinib+icotinibfor 3 months, PD. (C) 2019.5, alectinib+osimertinibfor 2 months, PD. (D) 2019.8, brigatinib+bevafor 2 months, PR. (E) 2020.2, loratinibfor 2 months, SD. 

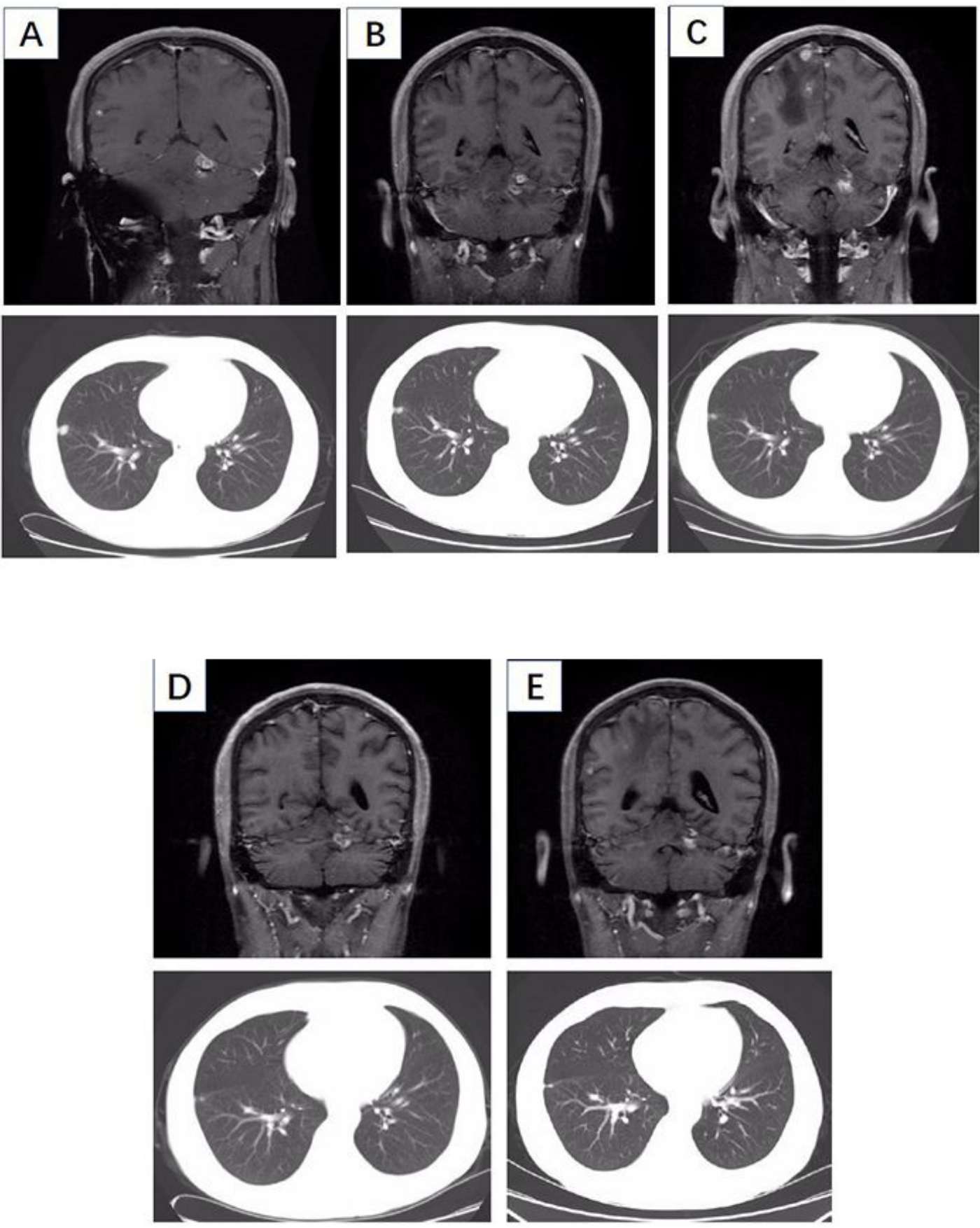

\section{Figure 2}

Serial MRI and CT of case 2. (A) 2018.7 Before crozitinib; (B) 2018.9 crozitinibfor 2 months, SD; (C) 2019.3 crozitinibfor 8 months, PD; (D) 2019.10 brigatinib+ icotinibfor 5months, PR; (E) 2020.07 brigatinib+ icotinibfor 11 months, PD. 

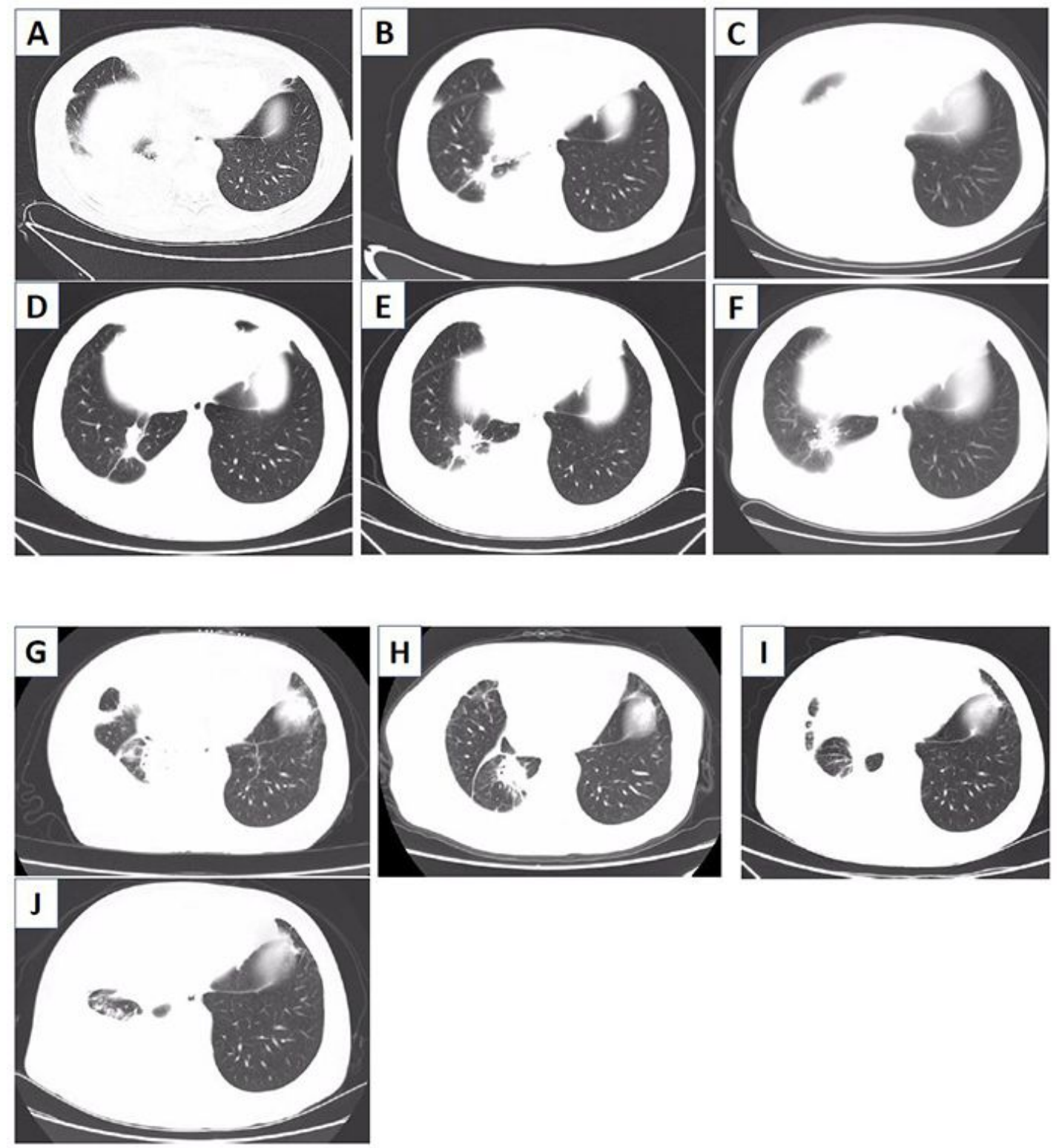

\section{Figure 3}

Serial CT of case 3. (A) 2015.4, baseline; (B) 2016.7, gefitinib for 15 months, PR; 2016.11, gefitinib for 19 months, PD; (D) 2017.7, AZD9291 for 8 months, PR; (E) 2017.11, AZD 9291 for 11 months, PD; (F) 2018.3, AZD 9291 + crozitinibfor 2 months, SD;(G) 2018.7, AZD9291 + crozitinibfor 7 months, PD; (H) 2019.1, Pem+ bevafor 7 cycles, SD; (I) 2020.04, before take osimertinib, PD; (J) 2020.08, Osimertinib for 4 months, PD. 


\section{Image not available with this version}

\section{Figure 4}

Univariant analysis showed that PFS have no significant difference $(p=0.132)$ (Fig 4a.). Among patients who were treated with EGFR-TKI as first-line targeted therapy, the median PFS was 7.0 months $(95 \% \mathrm{Cl}$ 0.75 - 13.25 months) and 7.7 months ( $95 \% \mathrm{Cl} 4.08$ - 11.32 months) in male and female (Fig 4b.), and was 6.0 months ( $95 \% \mathrm{Cl} 2.91-9.10$ months) and 7.0 months (95\% Cl $0.53-13.48$ months) in Asian and Caucasian (Fig 4c.). We can draw a conclusion that there was no statistically significant difference between male and female $(p=0.22)$ as well as Asian and Caucasian ( $=0.939)$. Additionally, the median PFS between first- and second-line targeted therapies was 7.0 months ( $95 \% \mathrm{Cl} 4.83-9.17$ months) and 2.0 months (95\% $\mathrm{Cl}$ 0.96-3.05 months), which showed no statistically different between these two groups ( $\mathrm{p}$ $=0.075$ ) (Fig 4d.). Survival curves showed the significantly prolonged PFS between patients without and with CNS metastases ( $p=0.036)$, which median PFS was 8.0 month ( $95 \% \mathrm{Cl} 0.53-13.48$ months) and 2.0 month ( $95 \% \mathrm{Cl} 0.53-13.48$ month) (Fig.4e). Only 14 patients can get the data of OS while the other missing data were not available, the average OS was 56.18 months ( $95 \% \mathrm{Cl} 32.72-72.9$ months) (Fig.4f).

\section{Supplementary Files}

This is a list of supplementary files associated with this preprint. Click to download.

- Table1.pdf

- Table2.pdf

- Table3.pdf 